\title{
Parâmetros Nutricionais em Pacientes Oncológicos atendidos em um Centro de Referência no Sul de Minas Gerais, Brasil
}

doi: https://doi.org/10.32635/2176-9745.RBC.2018v64n2.74

\author{
Nutritional Parameters in Patients with Cancer attended at a Reference Center in the south of Minas Gerais state, Brazil \\ Parámetros Nutricionales en Pacientes Oncológicos asistidos en un Centro de Referencia en el Sur de Minas Gerais, Brasil
}

\author{
Ana Cláudia Lucas Mezavila Carvalho'; Patricia Calori Martins²; Rhanna Bueno Araujo3; Cláudio Daniel Cerdeira4; Roberta Bessa \\ Veloso Silva5; Gérsika Bitencourt Santos Barros ${ }^{6}$
}

Resumo

Introdução: A orientação nutricional é de extrema importância para pacientes oncológicos, prevenindo deficiências nutricionais que podem gerar sérias complicaçóes. Objetivo: Avaliar o perfil nutricional de pacientes oncológicos. Método: Estudo transversal, realizado em um Centro de Referência em Oncologia de Alfenas - MG. Para traçar o perfil nutricional de 52 pacientes oncológicos ( $\mathrm{n}=52$ ) durante o tratamento quimioterápico, foram utilizados métodos dietéticos e antropométricos (índice de massa corporal [IMC], prega cutânea tricipital [PCT], circunferência do braço [CB], circunferência muscular do braço [CMB] e percentagem da perda de peso [\%PP]). Dados gerais de saúde dos pacientes foram também avaliados. Resultados: Houve predominância do sexo feminino (63\%), faixa etária >50 anos (40\% IC95\% 27-53,7). O tipo de câncer correlacionou-se ao sexo ( $\mathrm{p}<0,01)$. No sexo feminino, o de mama teve prevalência de $51 \%$, seguido pelo uterino (18\%). No masculino, a prevalência de câncer de próstata foi de 10\% e, comum aos dois sexos, o câncer de pulmão, de $15 \%$, sendo o mais prevalente no masculino (32\%). Hipertensão arterial sistêmica foi a comorbidade mais reportada (75\%) e enjoos, o efeito adverso mais comum (69\%). As médias dos parâmetros IMC, PCT, CB e CMB não sofreram alteraçóes significativas ( $\mathrm{p}>0,05)$ ao final do tratamento, mas $40 \%$ dos pacientes tiveram um grave $\% \mathrm{PP}, 23 \%$ não grave $\% \mathrm{PP}$, 4\% mantiveram o peso e $33 \%$ apresentaram ganho de peso. Entre os pacientes avaliados, $48 \%$ usavam suplementos nutricionais. Conclusáo: A orientação nutricional deve ser desenvolvida junto aos pacientes oncológicos, desde que se demonstrou um variado perfil nutricional em uma amostra heterogênea de pacientes.

Palavras-chave: Câncer; Quimioterapia; Índice de Massa Corporal; Hipertensão.

\section{Abstract}

Introduction: Nutritional counseling for patients with cancer is very important, because it can prevent nutritional deficiencies and other serious complications. Objective: To evaluate the nutritional profile of oncological patients. Method: Cross-sectional study, carried out at a reference center in Oncology, Alfenas, Minas Gerais, Brazil. Dietary and anthropometric methods (body mass index [BMI], triceps skinfold [TS], arm circumference $[\mathrm{AB}]$, arm muscle circumference $[\mathrm{AMC}]$, and percentage of weight loss [PWL]) were used to trace the nutritional profile of 52 oncological patients $(n=52)$ during chemotherapy. Other conditions of health were also evaluated. Results: There was a predominance of female sex (63\%), and the age group $>50$ years $(40 \%$ CI95[\%] 27-53.7). The type of cancer correlated with patient's sex $(\mathrm{p}<0.01)$. In female sex, the breast cancer had a prevalence of $51 \%$, followed by the uterus cancer (18\%). In male sex, the prevalence of prostate cancer was $10 \%$, and, common to both sexes, lung cancer had a prevalence of $15 \%$, being the most prevalent in males $(32 \%)$. Systemic arterial hypertension was the most reported comorbidity (75\%), and motion sickness the most common adverse event (69\%). The mean values of the parameters BMI, TS, AB and AMC did not change significantly ( $p>0.05)$ at the end of the treatment, but $40 \%$ of the patients had a severe PWL, 23\% no severe PWL, 4\% kept the weight, and 33\% presented weight gain. Among the patients evaluated, $48 \%$ used nutritional supplements. Conclusion: Nutritional counseling should be developed together with oncological patients, since we showed a variable nutritional profile in a heterogeneous sample of patients.

Key words: Cancer; Chemotherapy; Body Mass Index; Hypertension.

\section{Resumen}

Introducción: La orientación nutricional es de extrema importancia para los pacientes oncológicos, previniendo deficiencias nutricionales que pueden generar serias complicaciones. Objetivo: Evaluar el perfil nutricional de 52 pacientes oncológicos. Método: Estudio transversal, realizado en un centro de referencia en Oncología de Alfenas, Minas Gerais, Brasil. Para desenar el perfil nutricional de 52 pacientes oncológicos $(n=52)$ durante la quimioterapia, se utilizaron métodos dietéticos y antropométricos (índice de masa corporal [IMC], pliegue cutáneo tricipital [PCT], circunferencia del brazo [CB], circunferencia muscular del brazo $[\mathrm{CMB}]$ ) y el porcentaje de la pérdida de peso [\% $\mathrm{PP}])$. Se evaluaron también los datos generales de salud de los pacientes. Resultados: Hubo predominancia del sexo femenino (63\%), y la franja de edad $>50$ años (40\% IC95\% 27-53,7). El tipo de cáncer se correlacionó con el sexo ( $<<0,01)$. En el sexo femenino, el de mama tuvo prevalencia del 51\%, seguido por el uterino (18\%). Para el sexo masculino, la prevalencia de cáncer de próstata fue del $10 \%$ y, común a los dos sexos, el cáncer de pulmón tuvo una prevalencia del $15 \%$, siendo el más prevalente el sexo masculino (32\%). Hipertensión arterial sistémica fue la comorbilidad más reportada (75\%), y mareo el efecto adverso más común (69\%). Los valores de los parámetros IMC, PCT, CB y CMB no sufrieron cambios significativos $(\mathrm{p}>0,05)$ al final del tratamiento, pero el $40 \%$ de los pacientes tuvieron una grave $\% \mathrm{PP}$, el $23 \%$ una no grave $\% \mathrm{PP}$, el $4 \%$ mantuvieron el peso y el $33 \%$ de los evaluados presentaron una ganancia de peso. Entre los pacientes evaluados, $48 \%$ usaban suplementos nutricionales. Conclusión: La orientación nutricional debe desarrollarse junto a los pacientes oncológicos, una vez que se haya demostrado un variado perfil nutricional en una muestra heterogéneo de pacientes.

Palabras clave: Cáncer; Quimioterapia; Índice de Masa Corporal; Hipertensión.

\footnotetext{
1 Graduanda em Nutrição pela Faculdade de Nutrição da Universidade José do Rosário Vellano (Unifenas). Alfenas (MG),Brasil. E-mail:ana_claudialucasmezavila@ hotmail.com. Orcid ID: https://orcid.org/0000-0003-3352-1067

${ }^{2}$ Graduanda em Nutrição pela Faculdade de Nutrição da Unifenas. Alfenas (MG), Brasil. E-mail: patriciac.martins_alfenas@hotmail.com. Orcid ID: https://orcid. org/0000-0002-6263-2789

${ }^{3}$ Graduanda em Nutrição pela Faculdade de Nutrição da Unifenas. Alfenas (MG), Brasil. E-mail: rhanna_bueno_a@outlook.com. Orcid ID: https://orcid.org/00000002-9711-9520

${ }^{4}$ Doutorando em Ciências Farmacêuticas pelo Instituto de Ciências Biomédicas (ICB) da Unifal. Professor da Unifenas. Alfenas (MG), Brasil. E-mail: claudio. cerdeira@unifenas.br. Orcid ID: https://orcid.org/0000-0002-7242-8028

${ }^{5}$ Doutora. Professora da Unifenas. Alfenas (MG), Brasil. E-mail: bessaveloso@yahoo.com.br. Orcid ID: https://orcid.org/0000-0003-4794-5872

${ }^{6}$ Doutora. Professora da Unifenas. Alfenas (MG), Brasil. E-mail: gersika.santos@unifenas.br. Orcid ID: https://orcid.org/0000-0003-0849-2786

Endereço para correspondência: Cláudio Daniel Cerdeira: DBq, ICB, Unifal. Rua Gabriel Monteiro da Silva, 700, prédio E, Sala 207 C. Alfenas, MG, Brasil. CEP 37130-000.
} 


\section{INTRODUÇÃO}

Estimativas da Organização Mundial da Saúde (OMS) para 2030 apontam a ocorrência de 27 milhóes de novos casos de câncer e 75 milhóes de pessoas vivendo com a doença ao redor do mundo, com aproximadamente oito milhóes de óbitos, sendo 70\% em países em desenvolvimento. No Brasil, para o biênio 2018-2019, estima-se uma incidência de aproximadamente $600 \mathrm{mil}$ casos de câncer ${ }^{1}$.

De acordo com o Instituto Nacional de Câncer José Alencar Gomes da Silva (INCA), câncer é o nome dado a um conjunto de mais de 100 doenças, todas caracterizadas por um crescimento desordenado de células que invadem tecidos e órgãos, podendo atingir outros sítios do corpo, na chamada metástase ${ }^{1}$. Fatores ambientais e genéticos contribuem para a gênese do câncer, e as estratégias terapêuticas são: radioterapia, quimioterapia e cirurgia, ou uma combinação destas. Os agentes quimioterápicos podem causar efeitos colaterais, incluindo anorexia, enjoo, vômitos, estomatite, diarreia e necrose da mucosa do colón, comprometendo o estado nutricional do paciente ${ }^{2,3}$.

O câncer e as síndromes paraneoplásicas, bem como os efeitos colaterais do tratamento quimioterápico e radioterápico, podem causar alteraçôes metabólicas, prejudicar a ingestão alimentar, comprometer o aporte nutricional e, consequentemente, causar desnutrição no paciente. Nesse sentido, destaca-se a orientação nutricional como uma importante estratégia de tratamento não farmacológico para prevenir e minimizar os sintomas ocasionados pela doença e/ou tratamento ${ }^{2-9}$, contribuindo significativamente para a promoção de saúde e melhorias na qualidade de vida do paciente oncológico. Portanto, o presente estudo verificou o perfil nutricional de pacientes oncológicos atendidos em um Hospital do Sul de Minas Gerais, Brasil, visando a identificar as principais alteraçóes nutricionais e de saúde durante e após a quimioterapia antineoplásica.

\section{MÉTODO}

Trata-se de um estudo transversal, realizado em um Centro de Referência em Oncologia de Alfenas, Sul de Minas Gerais, Brasil. Como critérios de inclusão, a amostra constitui de pacientes de ambos os sexos, acima de 18 anos de idade, com diagnóstico de câncer, e submetidos a seçôes de quimioterapia antineoplásica. A coleta de dados foi realizada, no período de setembro de 2017 a fevereiro de 2018, por pesquisadores capacitados por meio de um estudo-piloto, envolvendo $10 \%$ do tamanho amostral final, e os resultados das aferiçóes foram comparados por meio da estatística kappa (grau de concordância entre os avaliadores $>0,8)$. Foram excluídos do estudo indivíduos edemaciados, indivíduos sem capacidade de ambulação e aqueles que estavam em uso de dieta enteral.

O presente trabalho foi previamente aprovado pelo Comitê de Ética em Pesquisa da Universidade José do Rosário Vellano (Unifenas-Alfenas), sob o número de protocolo CAAE: 73410817.2.0000.5143 e realizado mediante consentimento dos pacientes por escrito, após leitura e esclarecimentos dos propósitos da pesquisa e assinatura do Termo de Consentimento Livre e Esclarecido (TCLE).

Para traçar o perfil nutricional, foram utilizados métodos antropométricos e dietéticos. $\mathrm{Na}$ avaliação antropométrica, as medidas utilizadas para a avaliação foram: índice de massa corporal (IMC) e prega cutânea tricipital (PCT), que demonstram a reserva de gordura corporal; circunferência do braço (CB), representando o somatório do tecido ósseo, muscular e gorduroso; e circunferência muscular do braço $(\mathrm{CMB})$, que indica o comprometimento do tecido muscular. O peso corporal foi medido utilizando-se a balança antropométrica digital/ eletrônica (Ramuza).

O peso corporal no início do tratamento foi verificado no prontuário do paciente. Assim, foi obtida a percentagem da perda de peso (\%PP), calculada pela fórmula: Peso Atual - Peso Habitual/Peso habitual X 100, sendo considerada perda significativa maior ou igual a $5 \%$ de perda de peso em um mês; maior ou igual a 7,5\% em três meses; e maior ou igual a $10 \%$ em seis meses.

A estatura foi avaliada por meio do prontuário médico. Com os dados de peso e estatura, foi calculado o IMC, que consiste no peso em $\mathrm{Kg}$, dividido pelo quadrado da estatura em metros. Para a mensuração PCT, primeiramente, utilizou-se uma fita métrica em fibra de vidro (1,5 m, Worker) para medir a CB e, a partir disso, utilizando um adipômetro clínico, com precisão de aproximadamente $1 \mathrm{~mm}$, determinou-se a PCT.

Foram feitas três medidas no braço não dominante, considerando como valor final a média das três. A partir dessa medida, foi calculada a CMB. Nessa avaliaçáo antropométrica, a classificação do estado nutricional para PCT, $\mathrm{CB}$ e CMB seguiu os padróes propostos em literatura ${ }^{10,11}$. Os dados relacionados à história clínica dos pacientes foram obtidos nos prontuários médicos. A pressão arterial $(\mathrm{PA}, \mathrm{mmHg})$ foi aferida durante o acompanhamento. Todos os parâmetros foram avaliados durante e após a quimioterapia antineoplásica. Em razão dos diferentes protocolos clínicos associados a esses tratamentos, o tempo médio de tratamento foi estimado em 90 dias.

Os dados são apresentados como valores absolutos, percentagens e/ou média \pm desvio-padrão (DP). Para a comparação das médias, foi utilizado o teste t de Student 
para duas amostras pareadas (dependentes), ao nível nominal de 5\% de significância. A estatística multivariada foi utilizada para verificar a correlação entre as variáveis simultaneamente, a partir da técnica de componentes principais $^{12}$. O teste exato de Fisher ao nível de 5\% de significância foi usado para avaliar a independência entre duas variáveis qualitativas. Essas análises foram realizadas no software $\mathrm{R}$ ( $R$ Core Team, 2017). Um intervalo de confiança de $95 \%\left(\mathrm{IC}_{95 \%}\right)$ foi construído utilizando o software BioEstat ${ }^{\circ}$ 5.0).

\section{RESULTADOS}

Os dados demográficos e as condiçóes clínicas/ nutricionais na amostra avaliada no presente estudo são apresentados na Tabela 1 . Houve predominância de pacientes oncológicos do sexo feminino (63\%), com uma média de idade de aproximadamente 56 anos. Quanto ao tipo de câncer, no sexo feminino, entre as 33 mulheres avaliadas, 17 apresentaram câncer de mama (prevalência de 51\%), seguido por seis com câncer do útero (prevalência de $18 \%$ ). No sexo masculino, a prevalência de tumor de próstata foi $10 \%$. Em comum aos dois sexos, o câncer de pulmáo foi o mais observado (8 pacientes, 6 do sexo masculino), com uma prevalência geral de $15 \%$ e sendo o mais prevalente no sexo masculino (32\%). Os tipos de câncer diferiram em relação ao sexo $(p<0,01)$.

Todos os pacientes avaliados estavam em quimioterapia, considerando uma frequência de $53 \%$ dos pacientes com ciclo quinzenal e $47 \%$ com o semanal. O tempo médio de tratamento, até o momento da coleta de dados, foi de cinco meses. O perfil de medicamentos apresentados na Tabela 1 é relativo ao tratamento quimioterápico, bem como a terapia paliativa dos efeitos adversos da quimioterapia (como a ranitidina, indicada para úlceras estomacais; difenidramina, um anti-histamínico usado para tratamento de reaçóes alérgicas; e ondansetrona, usado para o alívio de enjoos e vômitos, relacionados à quimioterapia) e/ou para tratar outras doenças (como a cefalotina, um antibiótico), visto que apenas cinco pacientes náo apresentavam comorbidades.

Quanto à dieta dos pacientes, apenas 1,92\% apresentou restrição alimentar, enquanto $48,08 \%$ utilizavam algum tipo de suplementação energética (Tabela 1). Os resultados da avaliação antropométrica são observados na Figura 1, com apresentaçẫo do IMC (Figura 1 A), CB (Figura 1 B), classificação da CMB (Figura $1 \mathrm{C}$ ), adequação da PCT (Figura 1 D), e \%PP (Figura 1 E). Quanto ao \%PP, 40\% dos pacientes tiveram uma perda grave, $23 \%$ tiveram perda não grave e apenas $4 \%$ mantiveram o peso durante o tratamento. Trinta e três por cento dos avaliados apresentaram ganho de peso ao final do estudo.
$\mathrm{Na}$ Tabela 2, observa-se que não houve diferença significativa entre as médias durante e após o tratamento, para os parâmetros antropométricos e dietéticos, bem como a PA $(p>0,05)$. Contudo, em geral, na Figura 2 , é observado que essas variáveis estão altamente correlacionadas umas com as outras; ou seja, o aumento de uma pode ser em virtude de um aumento na(s) outra(s), e vice-versa (avaliados pela estatística multivariada). Os dados obtidos durante e no final do tratamento, para os parâmetros antropométricos e dietéticos, evidenciaram alta correlação entre as variáveis, e as retas na mesma direção indicam que elas são diretamente proporcionais; isto é, o aumento em uma delas acarretará um aumento em todas as outras (Figura $2 \mathrm{~A}$ ). De nota, destaca-se a alta correlação entre a pressão sistólica (início, durante e final do tratamento) com essas variáveis, indicando que, quanto maior o IMC, a pressão arterial tende a aumentar e, consequentemente, espera-se um aumento em todas as outras. Na Figura 2 B, o mapa de observaçóes ilustra a distribuição dos 52 pacientes ao longo dos quatro quadrantes. Verificou-se que, aproximadamente, 45\% dos participantes encontram-se no primeiro e segundo quadrantes da direita, superior e inferior, respectivamente, e 55\% nos quadrantes da esquerda (no sentido horário, terceiro e quarto, inferior e superior, respectivamente). Isso indica que as variáveis analisadas foram mais frequentes em $45 \%$ dos participantes.

\section{DISCUSSÃO}

No presente estudo, assim como encontrado por Tartari et al. ${ }^{4}$, houve predominância de pacientes oncológicos do sexo feminino, com uma maior prevalência de câncer de mama, confirmando os dados reportados pelo INCA ${ }^{1}$, os quais evidenciam que, depois do câncer de pele náo melanoma, o de mama é o mais comum no Brasil e no mundo. Esse câncer apresenta uma crescente incidência após os 50 anos de idade, como demonstrado no presente estudo, em que a maioria das pacientes apresentava idade acima dessa faixa etária.

Neste estudo, observa-se um perfil amplo relacionado a comorbidades, a efeitos adversos relatados pelos pacientes e à farmacoterapêutica associada. Os sintomas relatados pelos entrevistados como constipaçáo, enjoo, vômito e diarreia podem estar relacionados à quimioterapia, de acordo com Marchry et al. ${ }^{8}$, sendo comum em todos os pacientes a ocorrência de pelo menos um desses sintomas. Isso pode prejudicar a ingestão de alimentos, comprometendo o status nutricional do paciente oncológico. Segundo Ravasco ${ }^{9}$, a maneira mais prática e, na maioria das vezes, mais eficaz de auxiliar na terapia nutricional de pacientes oncológicos é o uso 
Tabela 1. Dados demográficos e condições clínicas/nutricionais na amostra de pacientes oncológicos ( $\mathrm{n}=52$ ) atendidos em um centro de referência no Sul de Minas Gerais, Brasil, entre setembro de 2017 e fevereiro de 2018

\begin{tabular}{|c|c|c|c|c|c|c|}
\hline \multirow[t]{2}{*}{ Variáveis } & \multirow{2}{*}{ Sexo } & \multicolumn{2}{|c|}{$\begin{array}{c}\text { Masculino (M) } \\
(n=19,37 \% ; \\
\text { IC } 95 \% 23,5-49,6)\end{array}$} & \multicolumn{2}{|c|}{$\begin{array}{c}\text { Feminino (F) } \\
(n=33,63 \% ; \\
\text { IC95\% 50,4-76,5) }\end{array}$} & \multirow{2}{*}{$\begin{array}{c}\text { Total } \\
(n=52)\end{array}$} \\
\hline & & $n\left(\%^{\mathrm{B}}\right)$ & IC95(\%) & $n\left(\%^{\mathrm{B}}\right)$ & IC95(\%) & \\
\hline \multirow{3}{*}{ Faixa etária (anos) ${ }^{n s}$} & $18-50$ & $5(10 \%)$ & NA & $12(23 \%)$ & $11,6-34,5$ & 17 (33\%) \\
\hline & Faixa etária (anos) ${ }^{\text {ns }}$ & $14(27 \%)$ & $14,9-39$ & $21(40 \%)$ & $27-53,7$ & $35(67 \%)$ \\
\hline & Total & 19 (37\%) & & $33(63 \%)$ & & 52 (100\%) \\
\hline \multirow{13}{*}{ Tipos de câncer** } & Mama & 0 & NA & $17(33 \%)$ & $19,9-45,4$ & $17(33 \%)$ \\
\hline & Pulmão & $6(11 \%)$ & $2,9-20,2$ & $2(4 \%)$ & NA & $8(15 \%)$ \\
\hline & Reto & $3(5 \%)$ & NA & $4(8 \%)$ & NA & 7 (13\%) \\
\hline & Útero & 0 & NA & $5(10 \%)$ & NA & $5(10 \%)$ \\
\hline & Próstata & $2(4 \%)$ & NA & 0 & NA & $2(4 \%)$ \\
\hline & Esôfago & $2(4 \%)$ & NA & 0 & NA & $2(4 \%)$ \\
\hline & Intestino & $1(2 \%)$ & NA & $1(2 \%)$ & NA & $2(4 \%)$ \\
\hline & Tipos de câncer** & $1(2 \%)$ & NA & $1(2 \%)$ & NA & $2(4 \%)$ \\
\hline & Estômago & $2(4 \%)$ & NA & 0 & NA & $2(4 \%)$ \\
\hline & Fígado & $1(2 \%)$ & NA & $1(2 \%)$ & NA & $2(4 \%)$ \\
\hline & Orofaringe & $1(2 \%)$ & NA & 0 & NA & $1(2 \%)$ \\
\hline & Boca & 0 & NA & $1(2 \%)$ & NA & $1(2 \%)$ \\
\hline & Duodeno & $1(2 \%)$ & NA & 0 & NA & $1(2 \%)$ \\
\hline \multirow{5}{*}{ Efeitos colaterais ${ }^{n s}$} & Constipação & $10(19 \%)$ & $8,5-29,9$ & $14(27 \%)$ & $14,9-39$ & $24(46 \%)$ \\
\hline & Enjoo & $12(23 \%)$ & $11,6-34,5$ & $24(46 \%)$ & $32,6-59,7$ & $36(69 \%)$ \\
\hline & Diarreia & $2(4 \%)$ & NA & $3(6 \%)$ & NA & $5(10 \%)$ \\
\hline & Vômito & $6(11 \%)$ & $2,9-20,2$ & $4(8 \%)$ & NA & $10(19 \%)$ \\
\hline & Febre & $2(4 \%)$ & NA & 0 & NA & $2(4 \%)$ \\
\hline \multirow{6}{*}{ Comorbidades $^{\text {ns }}$} & HAS & $16(31 \%)$ & $18,2-43,3$ & $23(44 \%)$ & $30,7-55,7$ & 39 (75\%) \\
\hline & Nenhuma & 0 & NA & $5(10 \%)$ & NA & $5(10 \%)$ \\
\hline & $\mathrm{DM}$ & 0 & NA & $3(5 \%)$ & NA & $3(5 \%)$ \\
\hline & Depressão & 0 & NA & $3(5 \%)$ & NA & $3(5 \%)$ \\
\hline & Efeitos colaterais ${ }^{\mathrm{ns}}$ & 0 & NA & $3(5 \%)$ & NA & $3(5 \%)$ \\
\hline & Outros $^{A}$ & $1(2 \%)$ & NA & $7(13 \%)$ & $4,2-22,7$ & $8(15 \%)$ \\
\hline \multirow{12}{*}{$\begin{array}{l}\text { Medicamentos } \\
\text { utilizados }^{\text {ns }}\end{array}$} & Antiparasitário & 0 & NA & $3(5 \%)$ & NA & $3(5 \%)$ \\
\hline & Capecitabina & 0 & NA & $11(21 \%)$ & $10,1-32,3$ & $11(21 \%)$ \\
\hline & Ranitidina & $10(19 \%)$ & $8,5-29,9$ & $22(42 \%)$ & $28,9-55,7$ & $32(61 \%)$ \\
\hline & Ciclofosfamida & 0 & NA & $1(2 \%)$ & NA & $1(2 \%)$ \\
\hline & Difenidramina & $1(2 \%)$ & $\mathrm{NA}$ & $3(6 \%)$ & NA & $4(8 \%)$ \\
\hline & Gencitabina & $1(2 \%)$ & $\mathrm{NA}$ & $4(8 \%)$ & NA & $5(10 \%)$ \\
\hline & Comorbidades $^{\text {ns }}$ & $3(6 \%)$ & NA & $3(6 \%)$ & NA & $6(12 \%)$ \\
\hline & Etoposideo & $4(8 \%)$ & NA & $2(4 \%)$ & NA & $6(12 \%)$ \\
\hline & Carboplatina & $1(2 \%)$ & NA & $3(6 \%)$ & NA & 4 (8\%) \\
\hline & Fluorulacila & $2(4 \%)$ & NA & $2(4 \%)$ & NA & $4(8 \%)$ \\
\hline & Oxaliplatina & $1(2 \%)$ & NA & $1(2 \%)$ & NA & $2(4 \%)$ \\
\hline & Cefalotina & 0 & NA & $1(2 \%)$ & NA & $1(2 \%)$ \\
\hline \multirow{3}{*}{ Restrições na dieta ${ }^{\text {ns }}$} & Sim & $1(2 \%)$ & NA & 0 & NA & $1(2 \%)$ \\
\hline & Não & $18(35 \%)$ & $21,4-47,5$ & $33(64 \%)$ & $50,4-76,5$ & $51(98 \%)$ \\
\hline & Total & $19(37 \%)$ & & $33(63 \%)$ & & 52 (100\%) \\
\hline \multirow{3}{*}{$\begin{array}{l}\text { Uso de } \\
\text { suplementos } \\
\text { nutricionais }\end{array}$} & Sim & $13(25 \%)$ & $13,2-36,8$ & $12(23 \%)$ & $11,6-34,5$ & $25(48 \%)$ \\
\hline & Não & 6 (12\%) & $2,9-20,2$ & $21(40 \%)$ & $27-53,7$ & 27 (52\%) \\
\hline & Total & 19 (37\%) & & 33 (63\%) & & 52 (100\%) \\
\hline
\end{tabular}

Legendas: Em relaçáo à variável sexo: ${ }^{* *}$ Significativo ao nível nominal de $1 \%(p<0,01) ;{ }^{n s}$ náo significativo ao nível de $5 \%$ de significância $(p>0,05)$, segundo o teste exato de Fisher. HAS: hipertensão arterial sistêmica; DM: diabetes mellitus; A outros: hipotireoidismo $(n=2, \mathrm{~F})$, hepatite $(1, \mathrm{~F})$, bronquite $(1, \mathrm{M})$, asma $(1, \mathrm{~F})$,

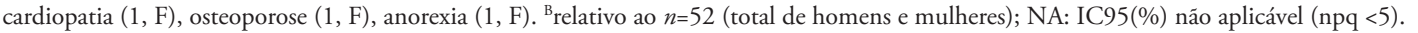


A.

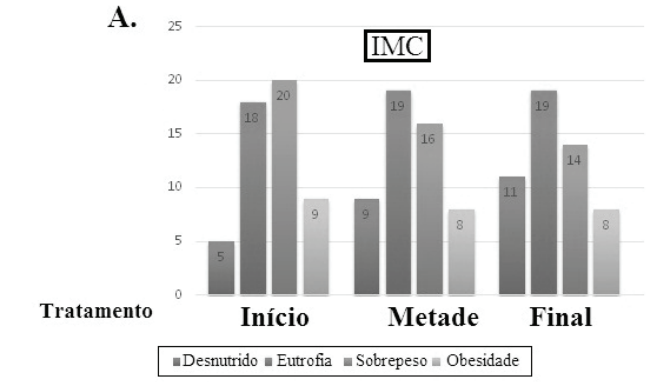

B.
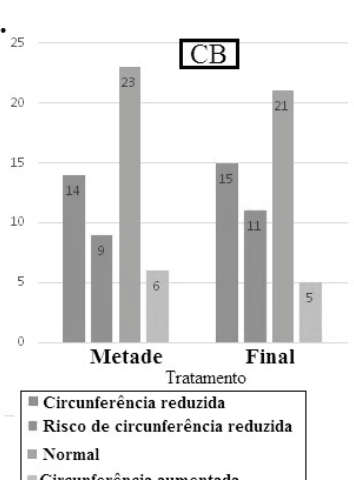

D.

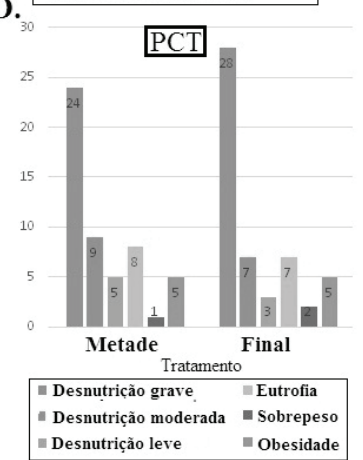

C.

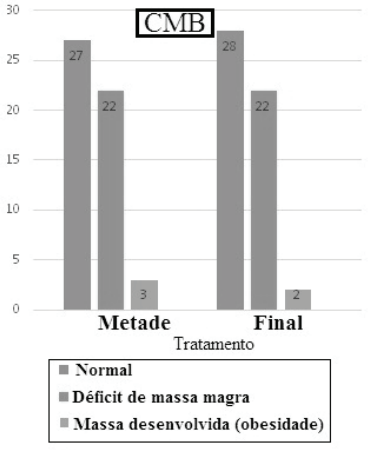

E.

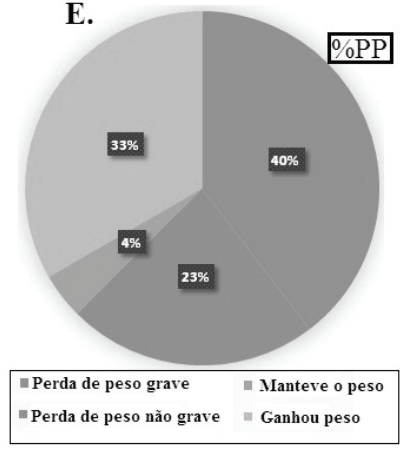

Figura 1. Número de pacientes oncológicos de acordo com os parâmetros antropométricos e dietéticos

A. Distribuição dos 52 pacientes oncológicos avaliados para o estado nutricional de acordo com IMC. B. Distribuição dos 52 pacientes oncológicos avaliados de acordo com a classificação da CB. C. Distribuição dos 52 pacientes oncológicos avaliados de acordo com a classificação da CMB. D. Distribuição dos 52 pacientes oncológicos avaliados de acordo com a adequação PCT. E. Percentual de pacientes oncológicos de acordo com a variação de peso durante tratamento Legendas: $\mathrm{IMC}=$ Índice de massa corporal; $\mathrm{CMB}=$ Circunferência muscular do braço; $\mathrm{CB}=$ Circunferência do braço; $\mathrm{PCT}$ = Prega cutânea tricipital; \%PP = Percentagem da perda de peso.

Valores de referência: IMC (Normalidade) $\rightarrow$ Faixa etária: 19 - 24 (19-24 kg/ $\left.\mathrm{m}^{2}\right) ; 25-34\left(20-25 \mathrm{~kg} / \mathrm{m}^{2}\right) ; 35-44\left(21-26 \mathrm{~kg} / \mathrm{m}^{2}\right) ; 45-54\left(22-27 \mathrm{~kg} / \mathrm{m}^{2}\right) ; 55-64$ $\left(23-28 \mathrm{~kg} / \mathrm{m}^{2}\right) ;>65\left(24-29 \mathrm{~kg} / \mathrm{m}^{2}\right)$ [Classificaçăo para idosos (> 60 anos): IMC $<22 \rightarrow$ Magreza; 22-27 $\rightarrow$ Eutrofia; $>27 \rightarrow$ Excesso de peso].

dos suplementos orais proteicos, que têm uma função importante quando há ingestão anormal de alimentos. No presente estudo, $48 \%$ dos pacientes oncológicos estavam em uso de suplementos orais, com a função de aumentar o ganho de peso e manter o paciente o mais nutrido possível. Também de acordo com Meyenfeldt ${ }^{13}$, o suplemento alimentar oral é a melhor escolha para uma
A.

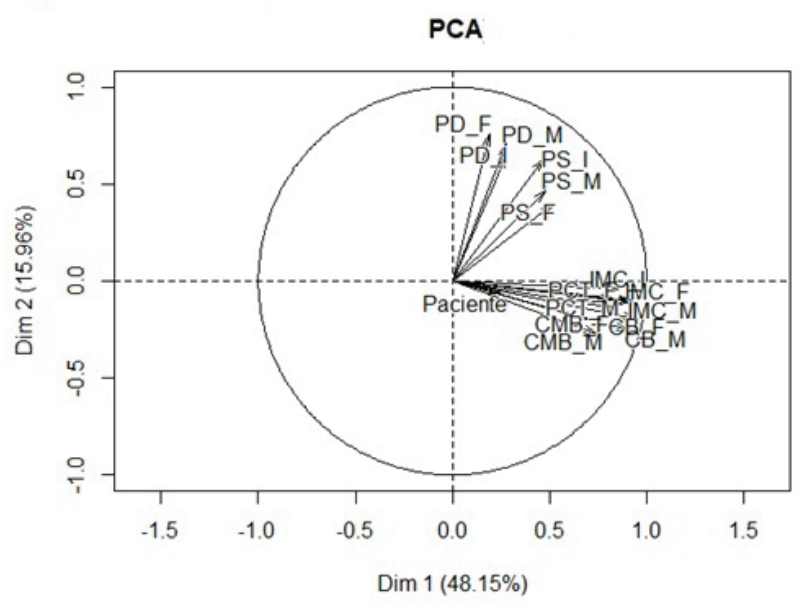

B.

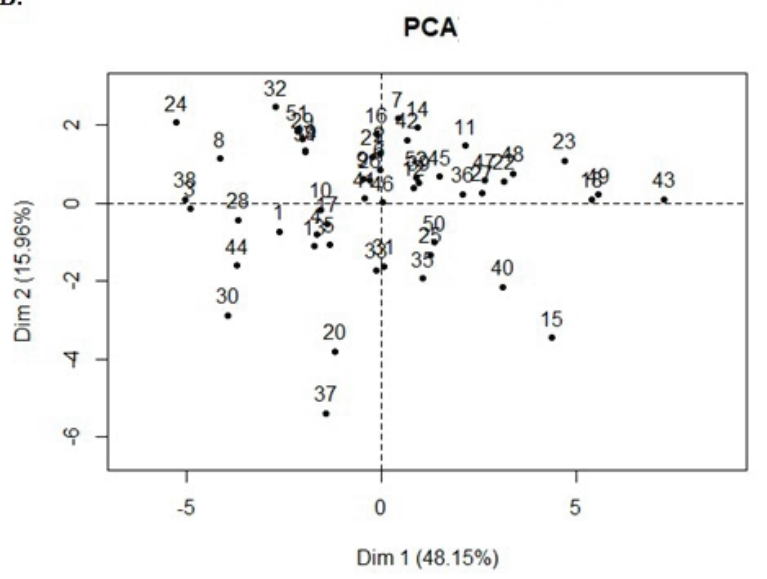

Figura 2. Mapas da estatística multivariada na amostra de 52 pacientes oncológicos

A. Mapa mostrando a correlação entre as variáveis analisadas. B. Mapa de observações mostrando a distribuição dos 52 participantes ao longo dos quatro quadrantes

Legendas: PS_I = Pressão arterial sistólica inicial; PS_M = Pressão arterial sistólica na metade do tratamento; PS_F = Pressão arterial sistólica final; PD_I = Pressão arterial diastólica inicial; PD_M = Pressão arterial diastólica na metade do tratamento; PD_F = Pressāo arterial diastólica final; IMC_I = Índice de massa corporal inicial; IMC_M = Índice de massa corporal na metade do tratamento; IMC_F: Índice de massa corporal final; PCT_I: Prega cutânea tricipital inicial; PCT_M = Prega cutânea tricipital na metade do tratamento; PCT_F = Prega cutânea tricipital final; CMB_I: Circunferência muscular do braço inicial; CMB _M = Circunferência muscular do braço na metade do tratamento; $\mathrm{CMB}$ _F = Circunferência muscular do braço final; $\mathrm{CB}_{-} \mathrm{I}=$ Circunferência do braço inicial; $\mathrm{CB} \_\mathrm{M}=$ Circunferência do braço na metade do tratamento; $\mathrm{CB} \_\mathrm{F}=$ Circunferência do braço final. intervençáo nutricional precoce, o que poderá aumentar a ingestão alimentar, sendo um método simples, natural e não invasivo. Argilés ${ }^{14}$ também corrobora a importância da intervenção nutricional por meio de orientaçôes sobre a dieta, uso de suplementos orais, a fim de prevenir ou tratar a desnutriçáo, aumentar os efeitos do tratamento e melhorar a qualidade de vida dos pacientes oncológicos. 
Tabela 2. Valores p obtidos por meio da comparação de médias durante e após o tratamento

\begin{tabular}{|c|c|c|c|}
\hline Comparações & Médias & $\begin{array}{l}\text { Desvio- } \\
\text {-padrão }\end{array}$ & Valor $p$ \\
\hline \multirow{2}{*}{$\begin{array}{l}\text { IMC durante } \\
\text { IMC final }\end{array}$} & 25,33 & 5,64 & \multirow{2}{*}{$0,7486^{n s}$} \\
\hline & 24,98 & 5,71 & \\
\hline \multirow{2}{*}{$\begin{array}{c}\text { CMB durante } \\
\text { CMB final }\end{array}$} & 22,44 & 3,49 & \multirow{2}{*}{$0,6233^{\text {ns }}$} \\
\hline & 22,12 & 3,29 & \\
\hline \multirow{2}{*}{$\begin{array}{l}\text { CB durante } \\
\text { CB final }\end{array}$} & & 27,63 & \multirow{2}{*}{$1,0000^{\text {ns }}$} \\
\hline & 27,13 & 4,98 & \\
\hline \multirow{2}{*}{$\begin{array}{l}\text { PCT durante } \\
\text { PCT final }\end{array}$} & 16,58 & 7,63 & \multirow{2}{*}{$0,6007^{n s}$} \\
\hline & 15,79 & 7,68 & \\
\hline \multirow{2}{*}{$\begin{array}{l}\text { PAS durante } \\
\text { PAS final }\end{array}$} & 120,59 & 10,54 & \multirow{2}{*}{$0,5460^{\text {ns }}$} \\
\hline & 119,42 & 9,16 & \\
\hline \multirow{2}{*}{$\begin{array}{l}\text { PAD durante } \\
\text { PAD final }\end{array}$} & 72,79 & 10,86 & \multirow{2}{*}{$0,7503^{n s}$} \\
\hline & 73,46 & 10,64 & \\
\hline
\end{tabular}

Legendas: ${ }^{n}$ náo significativo ao nível de $5 \%$ de significância $(p>0,05)$, de acordo com o teste $t$ de Student. IMC: índice de massa corporal; CMB: circunferência muscular do braço; $\mathrm{CB}$ : circunferência do braço; PCT: prega cutânea tricipital; PAS: pressão arterial sistólica; PAD: pressão arterial diastólica.

Valores de referência: IMC (normalidade) $\rightarrow$ faixa etária: 19-24 $\left(19-24 \mathrm{~kg} / \mathrm{m}^{2}\right)$; $25-34\left(20-25 \mathrm{~kg} / \mathrm{m}^{2}\right) ; 35-44\left(21-26 \mathrm{~kg} / \mathrm{m}^{2}\right) ; 45-54\left(22-27 \mathrm{~kg} / \mathrm{m}^{2}\right) ; 55-64(23-28$ $\left.\mathrm{kg} / \mathrm{m}^{2}\right) ;>65\left(24-29 \mathrm{~kg} / \mathrm{m}^{2}\right)$ [classificação para idosos ( $>60$ anos): IMC $<22 \rightarrow$ magreza; 22-27 $\rightarrow$ eutrofia; $>27 \rightarrow$ excesso de peso]; valores normais de PA $\rightarrow$ sistólica $\leq 140 \mathrm{mmHg}$ e diastólica $\leq 90 \mathrm{mmHg}$ (observação: a OMS estabelece que afetados pela hipertensão arterial sistêmica [HAS] apresentam PA sistólica $\geq 140 \mathrm{mmHg}$ ou diastólica $\geq 90 \mathrm{mmHg}$ ou săo hipertensos em tratamento).

Em geral, quanto mais agressivo o tratamento do câncer, mais rápida é a desnutrição associada, diminuindo a qualidade de vida do paciente. Como exemplos, Dias et al. ${ }^{2}$ relataram que a melhoria do estado nutricional aumenta a resposta do paciente submetido ao tratamento para o câncer e reduz os efeitos colaterais, adaptando-o melhor ao programa de reabilitação. Ainda, Scantz et al. ${ }^{15}$ e Maio et al. ${ }^{16}$ observaram um IMC diminuído em poucos pacientes avaliados, que pode ser pela ocorrência de retenção hídrica, degradação proteica, expansão de líquido extracelular e, portanto, mascarando o real estado nutricional do paciente oncológico. Em concordância com Scantz et al..$^{15} \mathrm{e}$ Maio et al. ${ }^{16}$, Azevedo et al..$^{5}$ confirmaram uma prevalência de IMC diminuído também em poucos pacientes, sugerindo que pacientes com câncer podem apresentar diminuição da massa celular e expansão de outros compartimentos. Contudo, Pelissaro et al. ${ }^{16}$ relataram que o IMC é um parâmetro de diagnóstico isolado, com diversas limitaçôes. No presente estudo, não houve diferenças significativas quanto ao IMC entre os períodos aqui avaliados. A média no começo do tratamento foi de $26,3 \mathrm{Kg} / \mathrm{m}^{2}$, indicando um sobrepeso; na metade do tratamento, foi de $25,3 \mathrm{Kg} / \mathrm{m}^{2}$ (classificado como sobrepeso); e, no final, foi de $24,9 \mathrm{Kg} / \mathrm{m}^{2}$ (indicando o limite da eutrofia). Esse IMC elevado no início pode estar relacionado ao tipo de câncer de maior prevalência, que, no presente estudo, foi o de mama, uma vez que a maior parte dos entrevistados com sobrepeso e obesidade apresentava câncer de mama. Tartari et al. ${ }^{4}$ reportaram uma associaçáo entre ganho de peso e IMC elevado em pacientes com câncer de mama. Kitynec et al. ${ }^{18}$ relataram que a causa desse ganho de peso não é clara, mas pode estar relacionada com a ingestáo alimentar, diminuiçáo da atividade física, alteração da taxa metabólica basal ou/e menopausa.

No presente estudo, observa-se uma frequência de perda de peso grave nos pacientes sob tratamento, de $40 \%$, o que poderia ser esperado, estando isso relacionado aos efeitos colaterais do tratamento, em que $98 \%$ dos entrevistados apresentavam manifestaçóes gastrointestinais. Esses dados estáo em conformidade com os achados de Dias et al. ${ }^{2}$, que encontraram uma prevalência de $55 \%$ de perda de peso, e a metade dos pacientes avaliados apresentou, juntamente com a perda de peso, manifestaçóes gastrintestinais e diminuição da ingestão energética, tornando maior o risco de desnutrição. Houve um considerável percentual de ganho de peso, durante o tratamento, podendo estar relacionado também à prevalência do tipo de câncer. Contudo, o excesso de peso pode estar associado a doenças crônicas não comunicáveis, como a HAS. Corroborando tal fato, assim como no estudo de Del Rio et $a .^{6}{ }^{6}$, na presente pesquisa, observou-se maior prevalência de HAS (75\%). Complementando esses achados, Souza et al. ${ }^{19}$ constataram que o uso de quimioterápicos e fármacos adjuvantes utilizados no tratamento do câncer aumenta a sobrevida do paciente, mas, como consequência, aumenta a incidência de HAS.

De acordo com o $\mathrm{CB}$, a maioria dos entrevistados apresentou circunferência normal tanto na metade do tratamento quanto no final e, com circunferência reduzida, foram classificados $28 \%$ dos entrevistados, diferente do estudo exposto por Tartari et al. ${ }^{4}$, em que a maior parte dos entrevistados apresentava risco nutricional e de desnutrição. Por meio da medida PCT, verificou-se que houve prevalência de desnutrição grave tanto na metade do tratamento quanto no final, com $53 \%$ de pacientes apresentando maior déficit nutricional comparado ao IMC, o qual apresentou menor número de pacientes desnutridos. No estudo de Garófolo et al. ${ }^{20}$, essa diferença também foi constatada, sugerindo que a PCT revela maior percentual de déficit nutricional quando comparada ao IMC. O peso isolado não reporta claramente o segmento corporal que está em déficit, como também reportado por Ikemori et al. ${ }^{21}$. Analisando os dados da CMB, houve prevalência de normalidade tanto na metade do tratamento quanto no final, diferente do apresentado por Lopez \& Petrilli et al. ${ }^{7}$, em que os pacientes apresentavam um sinal de depleçáo muito grave. No caso do presente estudo, o déficit de massa magra foi de $42 \%$ tanto na metade do tratamento quanto no final. 


\section{CONCLUSÃO}

Neste estudo, efeitos adversos provenientes da específica terapêutica quimioterápico-antineoplásica e de comorbidades foram observados, podendo influenciar o quadro nutricional dos pacientes oncológicos. Os parâmetros nutricionais aqui avaliados, individualmente, não mostraram significativas alteraçôes durante as referidas terapias. Contudo, quando esses dados são analisados em conjunto, observaram-se variaçóes de peso e estado nutricional, também correlacionados a outros parâmetros de saúde, com relevância para intervençóes nutricionais (como uso de suplementos e adequaçóes dietéticas), visando a evitar agravos de saúde, como a desnutriçáo, e melhorando a qualidade de vida do paciente oncológico.

\section{CONTRIBUIÇÕES}

Ana Cláudia Lucas Mezavila Carvalho e Patricia Calori Martins contribuíram no desenho do trabalho, coleta dos dados, na escrita e aprovação final. Rhanna Bueno Araujo participou da concepção do trabalho, coleta dos dados, escrita e aprovação final. Cláudio Daniel Cerdeira contribuiu no desenho do trabalho, análise e interpretação dos dados, escrita e aprovou a versão final. Roberta Bessa Veloso Silva participou do desenho do trabalho, análise estatística dos dados, escrita e aprovação final. Gérsika Bitencourt Santos Barros participou da concepção do trabalho, coleta dos dados, escrita e aprovação final.

\section{DECLARAÇÃO DE CONFLITO DE INTERESSES}

Nada a declarar.

\section{REFERÊNCIAS}

1. Instituto Nacional de Câncer José Alencar Gomes da Silva. Estimativa da incidência de câncer no Brasil. Rio de Janeiro: Ministério da Saúde; 2018.

2. Dias MV, Barreto APM, Coelho SC, Ferreira FMB, Vieira GBS, Cláudio MM, et al. O grau de interferência dos sintomas gastrintestinais no estado nutricional do paciente com câncer em tratamento quimioterápico. Rev Bras Nutr Clin.2006; 21(3):211-8.

3. Palmieri BN, Moulatlet EM, Buschinelli LKO, Silva MEM. Aceitação de preparaçôes e sua associação com os sintomas decorrentes do tratamento de câncer em pacientes de uma clínica especializada. Cad. saúde colet. 2013; 21(1):2-9.

4. Tartari RF, Busnello FM, Nunes CHA. Perfil nutricional de pacientes em tratamento quimioterápico em um ambulatório especializado em quimioterapia. RBC. 2010; 56(1):43-50.
5. Azevedo CD, Bosco SMD. Perfil nutricional, dietético e qualidade de vida de pacientes em tratamento quimioterápico. ConScientia e Saúde. 2011; 10(1):23-30.

6. Del Rio G, Zironi S, Valeriani L, Bondi M, Bertolini $\mathrm{M}$, Piccinini L, et al. Weight gain in women with breast cancer treated with adjuvant cyclophosphomide, methotrexateand 5-flourouracil. Analysis of resting energy expenditure and body composition. Breast Cancer Res Treat. 2002; 73(3):267-73.

7. Lopez FA, Petrilli AS, Garófolo A. Alta prevalência de desnutrição em pacientes com tumores sólidos náo-hematológicos medida pelas pregas cutâneas e circunferências. São Paulo Med J. 2005; 123(6):277-81.

8. Machry RV, Susin CF, Barros RC, Lago LD. Desnutrição em pacientes com câncer avançado: uma revisão com abordagem para o clínico. Revista da AMRIGS. 2011; 25(3):296-301.

9. Ravasco P. Nutrition in Cancer. Nestle Nutr Inst Workshop Ser. 2015; 82: 91-102.

10. Frisancho AR. New norms of upper limb fat and muscle areas for assessment of nutritional status. Am J Clin Nutr. 1981; 34(11):2540-2545.

11. Frisancho AR. Anthropometric standards for the assessments of growth and nutritional status. Ann Arbor: University of Michigan Press;1990.

12. Ferreira DF. Estatística multivariada. Daniel Furtado Ferreira. 2. ed. Lavras: Editora UFLA, 2011.

13. Meyenfeldt MV. Cancer-associated malnutrition: An introduction. Eur J Oncol Nurs. 2005; 9 (Supl.):535-8.

14. Argilés JM. Cancer-associated malnutrition. Eur J Oncol Nurs. 2005; 9 (Supl.):539-50.

15. Schantz SP, Zhang ZF, Spitz MS, Sum M, Hsu TC. Genetic susceptibility to head and neck cancer: interaction between nutrition and mutagen sensitivity. Laryngoscope. 1997; 107(6):765-81.

16. Maio R, Berto JC, Corrêa CR, Campana AO, Paiva SAR. Estado nutricional e atividade inflamatória no pré-operatório em pacientes com cânceres na cavidade oral e da orofaringe. RBC. 2009; 55(4):345-53.

17. Pelissaro E, Damo CC, Alves ALS, Loss SH, Antoniazzi JL, Steemburgo T et al. Avaliação do estado nutricional em pacientes idosos oncológicos internados em um hospital de alta complexidade do Norte do Rio Grande do Sul. Scientia Medica. 2016; 26(2):7.

18. Kutynec CL, McCargar L, Barr SI, Hislop TG. Energy balance in women with breast cancer during adjuvant treatment. J Am Diet Assoc. 1999; 99 (10):1222-7.

19. Souza VB, Silva EM, Ribeiro ML, Martins WA. Hipertensão arterial no paciente com câncer. Arq. Bras. Cardiol. 2015; 104(3):246-252.

20. Garófolo A, Lopez FA, Petrilli AS. High prevalence of malnutrition among patients with solid non- 
hematological tumors as found by using skinfold and circumference measurements. Sao Paulo Med J. 2005; 123(6):277-81.

21. Ikemori EHA, Oliveira T, Serralheiro IFD, Shibuya E, Trintin LA, Assaf L, et al. Nutrição em oncologia. São Paulo: Marina e Tecmedd; 2003.

Recebido em 11/7/2018

Aprovado em 23/8/2018 